\title{
Footwear for osteoarthritis of the lateral knee: protocol for the FOLK randomised controlled trial
}

\author{
Kade L. Paterson ${ }^{1 *}$, Kim L. Bennell ${ }^{1}$, Ben R. Metcalf ${ }^{1}$, Penny K. Campbell ${ }^{1}$, Jessica Kasza ${ }^{2}$, Tim V. Wrigley ${ }^{1}$ and
} Rana S. Hinman ${ }^{1}$

\begin{abstract}
Background: Structural features of lateral tibiofemoral (TF) joint osteoarthritis (OA) occur in up to half of all people with knee OA, and co-existing lateral TF OA is associated with worse knee pain in people with mixed compartmental knee OA. Clinical guidelines for management of knee OA advocate advice about appropriate footwear, yet there is no research evaluating which types of footwear are best for managing pain associated with lateral TF OA. Biomechanical evidence suggests that "motion-control" footwear, which possess midsoles that are stiffer medially compared to laterally, may shift load away from the lateral compartment of the knee and thus may reduce knee pain associated with lateral TF OA. The primary aim of this study is to compare the effects of motioncontrol shoes to neutral shoes on knee pain in people with predominantly lateral TF OA.
\end{abstract}

Methods: This will be an assessor- and participant-blinded, two-arm, comparative effectiveness randomized controlled trial $(\mathrm{RCT})$ conducted in Melbourne, Australia. We will recruit a minimum of 92 people with painful lateral TF OA from the community. Participants will be randomly allocated to receive either motion-control shoes or neutral shoes and will be instructed to wear their allocated shoes for a minimum of $6 \mathrm{~h}$ per day for 6 months. The primary outcome is change in self-reported knee pain on walking, measured using a numerical rating scale, assessed at baseline and 6 months. Secondary outcomes include other measures of knee pain, physical function, quality of life, participant-perceived change in pain and function, and physical activity levels.

Discussion: This study will compare the efficacy of motion-control shoes to neutral shoes for people with painful lateral TF OA. Findings will be the first to provide evidence of the effects of footwear on knee pain in this important subgroup of people with knee OA and allow clinicians to provide accurate advice about the most appropriate footwear for managing pain associated with lateral TF OA.

Trial registration: This trial has been prospectively registered by the Australian New Zealand Clinical Trials Registry on 15/11/2018 (reference: ACTRN12618001864213).

Keywords: Osteoarthritis, OA, Knee, Tibiofemoral, Footwear, Shoes, Clinical trial, RCT, Biomechanics, Pain

\footnotetext{
* Correspondence: kade.paterson@unimelb.edu.au

${ }^{1}$ Centre for Health, Exercise and Sports Medicine, Department of

Physiotherapy, School of Health Sciences, Faculty of Medicine Dentistry \&

Health Sciences, The University of Melbourne, Melbourne, Australia

Full list of author information is available at the end of the article
}

C C The Author(s). 2020 Open Access This article is licensed under a Creative Commons Attribution 4.0 International License, which permits use, sharing, adaptation, distribution and reproduction in any medium or format, as long as you give appropriate credit to the original author(s) and the source, provide a link to the Creative Commons licence, and indicate if changes were made. The images or other third party material in this article are included in the article's Creative Commons licence, unless indicated otherwise in a credit line to the material. If material is not included in the article's Creative Commons licence and your intended use is not permitted by statutory regulation or exceeds the permitted use, you will need to obtain permission directly from the copyright holder. To view a copy of this licence, visit http://creativecommons.org/licenses/by/4.0/ The Creative Commons Public Domain Dedication waiver (http://creativecommons.org/publicdomain/zero/1.0/) applies to the data made available in this article, unless otherwise stated in a credit line to the data. 


\section{Background}

Osteoarthritis (OA) is a leading cause of pain and the 12th highest contributor to global disability [1]. The knee joint is often affected, and abnormal knee joint loading is central to OA pathogenesis [2, 3]. During walking, the ground reaction force vector typically passes medially to the knee joint centre, resulting in an external knee adduction moment. The knee adduction moment is a valid indicator of medial-to-lateral tibiofemoral (TF) joint load distribution [4], and it is this imbalance of force towards the medial compartment during loadbearing that likely explains why the medial TF compartment is more frequently affected by OA than the lateral compartment [5]. Nonetheless, structural features of lateral TF joint OA occur in $10-55 \%$ of cases of knee OA [5-9], and research has shown that co-existing lateral TF OA is associated with worse knee pain in people with mixed compartmental knee OA [10].

Non-surgical biomechanical treatment strategies are often advocated [11], and used clinically, to manage people with TF OA. Footwear is a promising avenue for self-management, given that foot position and motion influence medial-to-lateral TF load distribution. Accordingly, international clinical guidelines recommend clinicians provide advice regarding appropriate footwear to help manage the symptoms of knee OA $[12,13]$. Unfortunately, all research evaluating footwear for OA has focussed on people with predominantly medial TF OA, and there are no RCTs evaluating the efficacy of footwear for people with predominantly lateral TF OA. This is a problem given that the biomechanics of people with lateral TF OA differ from those with medial TF OA [14], thus any evidence about the (in) effectiveness of biomechanical treatments for medial TF OA cannot be directly translated to the lateral TF OA. In support, leading international OA organisations have identified research on biomechanical interventions for specific OA subgroups as a key research recommendation [12].

In people with medial knee OA, a number of studies have shown that lateral wedge insoles [15], flat flexible shoes [16], and shoes with midsoles that are stiffer laterally than medially redistribute knee load away from the medial TF compartment towards the lateral compartment [17], albeit clinical effects are uncertain [18]. This is achieved via a lateral shift in the frontal plane ground reaction force-knee joint centre lever arm [19, 20] and an increase in lateral plantar pressures [21]. Likewise, footwear with midsoles that are stiffer medially compared to laterally, such as those with features that provide stability/support to the medial aspect of the foot, shift load toward the medial compartment [16, 22, 23], likely with a concomitant reduction in the lateral TF compartment. Such shoes are often referred to as "motion-control" or "stability" footwear. Although no study has directly investigated motion-control shoes, other research has shown that medially-wedged insoles, which also increase medial plantar pressures [24], reduce knee valgus malalignment in people with lateral knee OA [25]. In healthy populations, medially-wedged insoles [26] and medial arch supports [27] have also been shown to redistribute knee joint loading toward the medial TF compartment.

There is indirect RCT evidence supporting potential clinical improvements in people with lateral TF OA wearing motion-control footwear. A small RCT compared 30 women with lateral TF OA and bilateral knee valgus deformity wearing either medially wedged or neutral orthoses for 3-6 h/day for 8 weeks [28]. Compared to the neutral insoles group, the medially-wedged orthoses group showed greater improvements in pain with movement over 8 weeks, measured using a visual analogue scale (VAS; medially-wedged insoles: $-49 \%$ vs neutral insoles: $-6 \%$, between group change $P=0.001$ ), and in the Western Ontario McMasters University Osteoarthritis Index (WOMAC) total score $(-25 \%$ vs $3 \%, P=$ 0.001). Improvements in pain with movement and the total WOMAC score in the medially-wedged insole group exceeded minimal clinically important differences. However, the results of this small trial of medial wedge orthoses inserted into female participant's own usual footwear cannot necessarily be extrapolated to shoes with in-built motion control features. There have been no RCTs testing the efficacy of any type of footwear on symptoms in people with lateral TF OA. As such, the efficacy of motion-control shoes for this condition remains unknown, and there is no evidence to inform clinical guidelines about which type of footwear is best for this important subgroup of patients with knee OA.

This study outlines the protocol for a RCT of footwear for people with symptomatic radiographic lateral TF OA. The primary aim of this RCT is to compare the effects of motion-control shoes and neutral shoes on knee pain in people with lateral TF OA. It is hypothesised that motion-control shoes will lead to significantly greater reductions in knee pain with walking compared to neutral shoes, when worn daily over 6 months, in people with lateral TF OA. Our secondary aim is to assess whether motion-control shoes improve other measures of pain, function, quality of life and physical activity compared to neutral shoes.

\section{Methods}

\section{Study design}

This study is a participant- and assessor-blinded, twoarm, comparative effectiveness RCT. The trial is being conducted at The University of Melbourne. It was prospectively registered with the Australian and New Zealand Clinical Trials Registry (ACTRN12618001864213) 
and is described using the Standard Protocol Items: Recommendations for Intervention Trials (SPIRIT) [29].

\section{Participants}

Participants with painful predominantly lateral TF OA are being recruited from the community using print, radio, and social media advertisements, and via our existing network of clinicians and our volunteer database. The American College of Rheumatology clinical and radiographic criteria is being used to classify participants as having knee OA [30]. Participants are eligible for the study if they meet the following inclusion criteria:

i) aged $\geq 50$ years;

ii) report knee pain on most days of the past month;

iii) report a minimum pain score of 4 on an 11-point numeric rating scale (NRS, with terminal descriptors of 'no pain' and 'worst pain possible') on average during walking over the previous week;

iv) have mild, moderate or severe (Grade 2-4) TF OA on $\mathrm{x}$-ray according to the Kellgren \& Lawrence (KL) grading system [31], one of the most widely used system for classifying radiographic severity of knee OA [32]; and

v) demonstrate a grade of lateral TF joint space narrowing greater than medial TF joint space narrowing, determined using a radiographic atlas [33] (where Grade $0=$ no narrowing, $1=$ mild narrowing, 2 = moderate narrowing, $3=$ severe narrowing).

Participants will be excluded if they:

i) report knee pain for $<3$ months;

ii) report recent knee surgery (in the past 6 months) or are planning to undergo surgery in the next 6 months;

iii) currently use foot orthoses, customised shoes, or ankle/knee braces;

iv) currently wear high heels, thongs or work boots for an extended period of time that would restrict their ability to wear the allocated study shoes for a minimum of $6 \mathrm{~h}$ per day;

v) have had a hip or knee replacement on their most painful side/knee;

vi) have had a high tibial osteotomy on their most painful knee;

vii) have had any injections in the knee joint in the past 3 months, or are planning an injection in next 6 months;

viii)report any other muscular, joint or neurological condition affecting lower limb function;

ix) report any systemic or inflammatory joint disease (eg rheumatoid arthritis); $x)$ currently use a gait aid, or plan to use one in the next 6 months;

xi) cannot understand written and/or spoken English;

xii) have a foot size outside the range of 6 to 12US for women and 7 to 13US for men; or

xiii)are unable to commit to the study requirements, such as wearing the study shoes, attending study appointments, or completing outcome measures.

\section{Procedure}

All potential participants receive oral and written information about the purposes, potential risks and processes involved in the study from the Trial Coordinator. Informed consent is obtained from all participants by signing the consent form after the plain language statement has been read, and the salient information delivered verbally, and before proceeding with radiographic eligibility screening. Ethical approval has been obtained from the University of Melbourne Human Research Ethics Committee (HREC No. 1852787.1).

The flow of participants through the study is outlined in Fig. 1. Potential volunteers are initially screened using an online form, and if they pass, are being contacted by the Trial Coordinator for further telephone screening. Those passing telephone screening are then booked for posteroanterior weightbearing $\mathrm{x}$-rays at one of three radiology clinics in Melbourne, Australia for screening against radiographic eligibility criteria. If a participant has had a weightbearing posteroanterior or anteroposterior $\mathrm{x}$-ray in the previous 2 years, and they are able to share the images with research staff for assessment, they are not required to undergo new $\mathrm{x}$-rays. X-rays are graded by experienced research staff to confirm eligibility. In case a participant has bilaterally eligible knees, the most symptomatic knee is considered the study knee.

Participants are completing baseline assessments at the Department of Physiotherapy, The University of Melbourne. Six-month follow-up assessments (surveys) are being completed either on paper or electronically at home. Participants are also required to record adherence with wearing their study shoes for 1 week per month over the intervention period, using log books. If a participant fails to complete or return a log book or the follow-up survey, a researcher contacts them by phone or email as a prompt. Every effort is being made to minimize loss of data, including collection of the primary outcome over the telephone if necessary.

\section{Randomisation, blinding and allocation concealment}

The randomisation schedule was generated by a biostatistician using permuted block sizes 6 to 12, and stratified by KL grades 2, 3 or 4. A researcher who is not involved with participant recruitment or assessment maintains the schedule on a password-protected website (REDCap) 


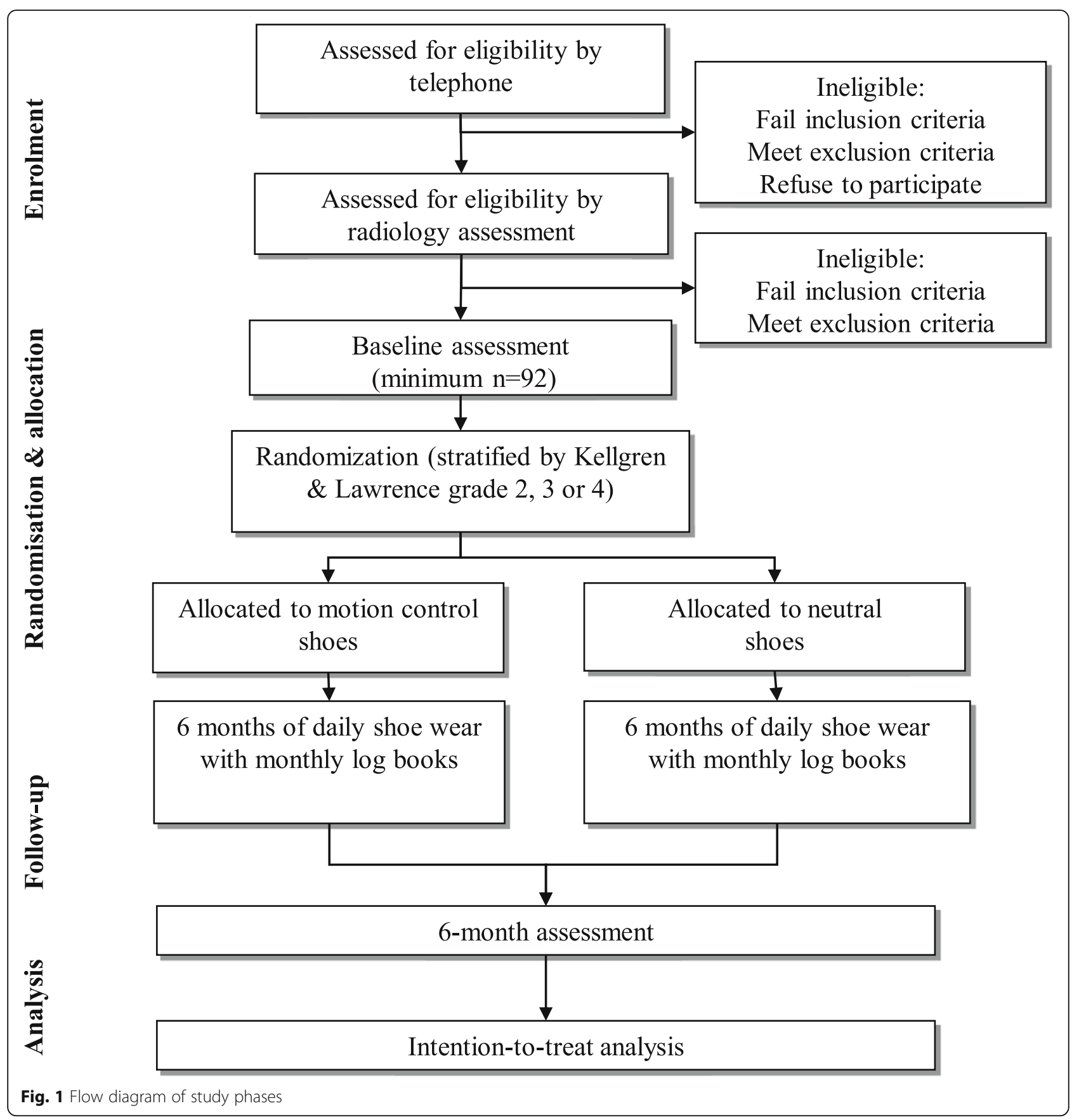

and reveals group allocation following baseline outcome assessment. This unblinded researcher is also responsible for measuring baseline participant characteristics in the laboratory, such as height, weight and foot posture, prior to fitting participants to their allocated shoes.

We use a process of limited disclosure to blind participants. We do not disclose any information to participants regarding the study hypotheses, or the shoe characteristics/model provided to participants in either group, to ensure blinding. Participants are told that we are comparing the effects of two types of readily available off-the-shelf walking shoes on knee OA symptoms in people with OA in the outer (lateral) compartment of the knee joint. Given participants are blinded to group allocation, and the primary and secondary outcomes are self-reported, this study is also considered assessor-blinded. Research staff administering and entering data, and the biostatistician performing statistical analyses, are also blinded.

\section{Footwear interventions}

Table 1 describes the intervention (motion-control) and the comparator (neutral) shoes. We are comparing two 
Table 1 Features of the intervention and comparator shoes

\begin{tabular}{|l|l|}
\hline Neutral shoes (comparator) & Motion-control shoes (intervention) \\
\hline $\begin{array}{l}\text { Gel-Nimbus (Asics Pty Ltd) } \\
\text { Mono-density midsole (i.e. similar medial and lateral } \\
\text { and bottom midsole layer, and rearfoot and forefoot gel. }\end{array}$ & $\begin{array}{l}\text { Gel-Kayano (Asics Pty Ltd) } \\
\text { Dual-density motion-control midsole that is stiffer } \\
\text { medially compared to laterally, and standard cushioning } \\
\text { features. }\end{array}$ \\
\hline
\end{tabular}

different shoe models from the same manufacturer (ASICS) that differ in the amount of support/stability provided to the medial aspect of the foot, but appear similar visually.

We have previously consulted people with knee OA ( $n=111$; 68 women and 43 men) about their shoe preferences for a clinical trial. This is to ensure we only test shoes that are acceptable to consumers, and that potential participants are willing to wear them for at least $6 \mathrm{~h}$ per day over 6 months. Using a survey format with photographs of different shoe options and colours, we presented a range of motion-control shoes to the consumer panel for consideration. Findings showed that the GelKayano (ASICS) shoes were a popular choice, and that black was overwhelmingly the preferred colour. Thus, we chose these as our intervention shoes. The predominant motion-control feature of the Gel-Kayano shoes is a dual-density 'motion-control' midsole that is stiffer medially compared to laterally. The comparator neutral shoe (Gel-Nimbus) was selected by the research team from other ASICS shoe models on the basis of being i) a neutral shoe with similar weight and structural characteristics to the intervention shoe, except for the dualdensity motion-control feature and; ii) similar in appearance to the intervention shoe to ensure participant acceptability and willingness to wear.

Participants are fitted with, and provided, a pair of their allocated shoes to take home. Participants are instructed to initially wear the shoes for $2 \mathrm{~h}$ on the first day only, and then to increase wear time by $2 \mathrm{~h} /$ day. We advise participants that they should be wearing their allocated shoes as much as possible every day by the end of the first week, aiming for a minimum of $6 \mathrm{~h} /$ day for 6 months. No study has investigated the minimum amount of time required to wear shoes to achieve a clinical effect for people with knee OA, therefore we chose $6 \mathrm{~h}$ as this was shown to be feasible in our previous knee OA footwear RCT [34], and likely provides enough time for a therapeutic effect.

\section{Outcome measures}

Table 2 describes the schedule of enrolment, interventions and the outcome measures for this study according to SPIRIT recommendations [29].

\section{Primary outcome}

Knee pain on walking Change in average pain on walking in the last week using an 11-point NRS with terminal descriptors 'no pain' (score $=0$ ) and 'worst pain possible' $($ score $=10)$ from baseline to 6 months. This is a valid and reliable OA outcome measure [35], and is recommended for knee OA clinical trials [36].

\section{Secondary outcomes}

Secondary outcomes are assessed at baseline and at 6 months unless indicated otherwise. These include:

\section{Physical function subscale of the WOMAC}

The Western Ontario and McMaster Universities (WOMAC) Osteoarthritis Index (Likert version 3.1) is used to assess limitations with physical functioning [37]. The self-reported tool is a disease-specific instrument which has established validity, reliability and responsiveness in an extensive range of OA studies [38]. The subscale contains 17 questions on knee function over the past week, with Likert response options from 'no dysfunction' (score $=0$ ) to 'extreme dysfunction' (score $=4$ ). Total score ranges from 0 to 68 , with higher scores indicating worse function. WOMAC scores will be extracted 
Table 2 Schedule of enrolment, interventions and assessments

\begin{tabular}{|c|c|c|c|c|c|c|c|c|}
\hline \multirow[b]{3}{*}{ TIMEPOINT } & \multicolumn{8}{|c|}{ STUDY PERIOD } \\
\hline & \multirow{2}{*}{$\begin{array}{c}\text { Enrolment } \\
-t_{1}\end{array}$} & \multirow{2}{*}{\begin{tabular}{|c|c|} 
Allocation \\
0
\end{tabular}} & \multicolumn{5}{|c|}{ Post-allocation } & \multirow{2}{*}{$\begin{array}{c}\text { Close-out } \\
6 m \\
\end{array}$} \\
\hline & & & $1 m$ & $2 m$ & $3 m$ & $4 m$ & $5 m$ & \\
\hline \multirow{4}{*}{$\begin{array}{r}\text { ENROLMENT: } \\
\text { Eligibility screen } \\
\text { Informed consent } \\
\text { Allocation }\end{array}$} & & & & & & & & \\
\hline & $\mathbf{X}$ & & & & & & & \\
\hline & $\mathbf{X}$ & & & & & & & \\
\hline & & $\mathbf{X}$ & & & & & & \\
\hline \\
\hline \multirow{2}{*}{\multicolumn{9}{|c|}{$\begin{array}{r}\text { Motion-control shoes } \\
\text { Neutral shoes }\end{array}$}} \\
\hline & & & & & & & & \\
\hline \multicolumn{9}{|l|}{$\begin{array}{l}\text { ASSESSMENTS: } \\
\end{array}$} \\
\hline \multicolumn{9}{|l|}{ Primary outcome } \\
\hline \multirow{2}{*}{$\begin{array}{l}\text { Knee pain on walking (11-point numerical scale) } \\
\text { Secondary outcomes }\end{array}$} & & $\mathbf{X}$ & & & & & & $\mathbf{X}$ \\
\hline & & & & & & & & \\
\hline Knee pain (KOOS pain subscale) & & $\mathbf{X}$ & & & & & & $\mathbf{X}$ \\
\hline \multirow{2}{*}{$\begin{array}{r}\text { Physical function (WOMAC physical function subscale) } \\
\text { Sport and recreation activities (KOOS sport and recreation subscale) }\end{array}$} & & $\mathbf{X}$ & & & & & & $\mathbf{X}$ \\
\hline & & $\mathbf{X}$ & & & & & & $\mathbf{X}$ \\
\hline Quality of life (KOOS quality of life subscale) & & $\mathbf{X}$ & & & & & & $\mathbf{X}$ \\
\hline Patellofemoral pain and OA (KOOS PFJ pain and OA subscale) & & $\mathbf{X}$ & & & & & & $\mathbf{X}$ \\
\hline Global improvement in pain & & & & & & & & $\mathbf{X}$ \\
\hline \multirow{4}{*}{$\begin{array}{r}\text { Global improvement in physical function } \\
\text { Health-related quality of life (AQoL-6D) } \\
\text { Physical activity (PASE) }\end{array}$} & & & & & & & & $\mathbf{X}$ \\
\hline & & $\mathbf{X}$ & & & & & & $\mathbf{X}$ \\
\hline & & $\mathbf{X}$ & & & & & & $\mathbf{X}$ \\
\hline & & & & & & & & \\
\hline \multirow{2}{*}{$\begin{array}{r}\text { Co-interventions } \\
\text { Adverse events }\end{array}$} & & $\mathbf{X}$ & & & & & & $\mathbf{X}$ \\
\hline & & & & & & & & $\mathbf{X}$ \\
\hline Adherence to wearing shoes & & & $\mathbf{X}$ & $\mathbf{X}$ & $\mathbf{X}$ & $\mathbf{X}$ & $\mathbf{X}$ & $\mathbf{X}$ \\
\hline \multirow{2}{*}{$\begin{array}{r}\text { Descriptive measures } \\
\text { Objective foot measures }\end{array}$} & & $\mathbf{X}$ & & & & & & \\
\hline & & $\mathbf{X}$ & & & & & & \\
\hline \multirow{2}{*}{$\begin{array}{r}\text { Usual footwear characteristics } \\
\text { Lower limb kinematics, kinetics and plantar pressures }\end{array}$} & & $\mathbf{X}$ & & & & & & \\
\hline & & $\mathbf{X}$ & & & & & & \\
\hline
\end{tabular}

WOMAC Western Ontario and McMaster Universities Osteoarthritis Index, KOOS Knee injury and osteoarthritis outcome score, PFJ Patellofemoral joint, OA Osteoarthritis, PASE Physical activity scale for the elderly, AQoL-6D Assessment of Quality of Life instrument (version 6D)

from the Knee Injury and Osteoarthritis Outcome Score (KOOS) questionnaire [39].

\section{Pain subscale of the KOOS}

The pain subscale of the KOOS is scored using nine questions regarding knee pain over the previous week, with Likert response options for each question ranging from none $($ score $=0)$ to extreme $($ score $=4)$ [39]. Scores are then transformed to provide an overall value that ranges from 0 to 100 , with 0 representing extreme knee pain and 100 representing no knee pain.

\section{Sport and recreation subscale of the KOOS}

The sport and recreation subscale is assessed using five questions on function during sport and recreational activities over the previous week [39]. Likert responses for each question range from none $($ score $=0)$ to extreme $($ score $=$ 4) [39]. Scores are then transformed to provide an overall value that ranges from 0 to 100 , with 0 representing extreme problems with sport and recreation and 100 representing no problems with sports and recreation.

\section{Quality of life subscale of the KOOS}

This subscale is assessed using four questions on kneerelated quality of life experienced in the previous week [39]. There are five Likert response options for each question, ranging from none $($ score $=0)$ to extreme $($ score $=4)$ [39]. Scores are then transformed to provide an overall value that ranges from 0 to 100 , with 0 representing extreme problems with quality of life and 100 representing no problems with quality of life. 


\section{Patellofemoral pain and OA subscale of the KOOS}

The patellofemoral pain and OA subscale includes 11 questions on knee pain and function experienced in the last week, each with five Likert response options, ranging from none $($ score $=0)$ to extreme $($ score $=4)$ [39]. Scores are then transformed to provide an overall value that ranges from 0 to 100 , with 0 representing extreme patellofemoral problems and 100 representing no patellofemoral problems.

\section{Participant-perceived global change}

Participants rate their overall global change in pain and change in physical function over the 6 months since commencing the study, using a 7-point Likert scale with terminal descriptors of 'much worse' to 'much better' [40]. Participants reporting that they are 'moderately better' or 'much better' are classified as improved.

\section{Health-related quality of life}

Health-related quality of life is evaluated using the Assessment of Quality of Life (AQoL) (version AQoL-6D) [41]. The AQoL-6D contains 20 items that assess independent living, mental health, relationships, pain, coping and senses. Total scores range from -0.04 to 1.00 , with higher scores indicating better quality of life.

\section{Physical activity levels}

Physical activity over the previous week is assessed using the Physical Activity Scale for the Elderly (PASE) [42]. Total PASE scores range from 0 to over 400, with higher scores indicating greater physical activity.

\section{Other measures}

Co-interventions Participants self-report co-intervention use (medications for knee pain and any other treatments for knee OA) at baseline and 6-months.

Adverse events Adverse events (any problem experienced in the study knee or elsewhere in the body as a result of wearing the study shoes) are self-reported by participants at 6 months, and the proportion experiencing adverse events described along with the nature of the adverse events.

Treatment adherence For each of 7 consecutive days each month of the 6-month intervention, participants record the number of hours that they wore their allocated shoes in log books. At the 6-month follow-up assessment, participants also rate their perceived overall level of adherence with wearing their allocated shoes (for a minimum of $6 \mathrm{~h}$ per day over the previous 6 months) on an 11-point NRS (with terminal descriptors of 'shoes not worn all' and 'shoes worn completely as instructed'). Finally, participants also indicate whether they stopped wearing their allocated shoes during the 6 months on a categorical scale (Yes or No) at the 6month follow-up. Participants who respond 'Yes' are required to describe when and why they stopped wearing their study shoes, and this will be reported descriptively.

Descriptive measures These are recorded at baseline, and include height, body mass and body mass index; age; gender; duration of symptoms; radiographic disease severity (measured using the KL scale [31]); anatomical knee alignment (measured from the knee $\mathrm{x}$-ray) [43]; current employment status; expectation of treatment outcome (rated on a 5-point ordinal scale with anchors of "no effect at all" to "complete recovery"); and selfefficacy (via the Arthritis Self Efficacy Scale [44]).

Objective foot measures Objective foot measures are assessed at baseline, including the Foot Posture Index (FPI [45]), Foot Mobility Magnitude [46], and navicular drop [47]. In-shoe regional foot pressure patterns (Novel Pedar, Munich, Germany) are assessed following randomisation whilst walking in usual footwear and allocated study shoes, in random order.

Usual footwear characteristics Motion-control characteristics of each participant's most commonly worn shoes are recorded at baseline using the relevant items of the Footwear Assessment Tool [16]. Number of participants and proportion of participants' usual shoes with motion-control features will be reported.

Lower limb kinematics and kinetics Participants are assessed following randomisation walking barefoot and in their allocated shoes, presented in random order, while 3-dimensional gait analysis is performed. Gait analysis will only be completed on participants with a BMI $<36 \mathrm{~kg} / \mathrm{m}^{2}$ due to difficulties performing gait analysis in people with a high BMI. A variety of biomechanical measures will be extracted, including parameters of the knee adduction/abduction moment (impulse and peaks). Changes in biomechanical parameters from the barefoot test condition to the allocated shoe condition will be compared between the two trial arms $[16,48])$.

\section{Sample size calculations}

We aim to detect the minimal clinically important difference on the primary outcome between groups (1.8 (out of 10) for NRS pain [49]). We conservatively assume a between-participant standard deviation of 2.7 units and a baseline to 6-month correlation of 0.21 based on our previous trials $[34,50]$. Using analysis of covariance adjusted for baseline score, we need a minimum of 46 per arm to achieve $90 \%$ power to detect the minimal 
clinically important difference in pain. Allowing for $15 \%$ loss to follow up, we aim to recruit 55 participants per group. However, due to slow recruitment of participants with lateral TF OA (given the lower prevalence of lateral compared to medial TF OA), we will recalculate the sample size based on an updated attrition rate after 92 participants have been enrolled in to the study. The proportion of participants who have provided the primary outcome of NRS pain during walking at 6 months when the 92nd participant is enrolled will be calculated (pooled across the study arms). The sample size will then be revised based on this new attrition rate: if $p$ patients are found to not have provided 6-month outcomes, the sample size required for each arm will be updated to $46 /(1-p)$.

\section{Statistical analyses}

A biostatistician will analyse blinded data. Main between-group comparative analyses will be performed using intention-to-treat, and multiple imputation will be used if more than $5 \%$ of the primary outcome is missing. Between-group differences in the mean change in the primary outcome of pain (baseline minus follow-up) will be compared using linear regression models, adjusted for baseline values of the primary outcome and the stratifying variable of KL grade. Continuous secondary outcomes will be analysed using similar methods. Risk differences, calculated from fitted logistic regression models, will be used to compare improvements in global change across groups. A sensitivity analysis will estimate treatment effects assuming full adherence to wear of the shoes (average of $6 \mathrm{~h} /$ day for 6 months, based on logbook data), using an instrumental variables approach [51]. Standard diagnostic plots will be used to check model assumptions.

To assess whether the effect of shoe allocation on the primary outcome is moderated by any of $\mathrm{KL}$ grade, FPI score, knee alignment, or baseline KOOS patellofemoral pain and OA score, appropriate interaction terms between randomised group and each of these variables will be included in regression models for the primary outcome, for each potential effect modifier separately.

\section{Timelines}

Ethics approval was obtained from the University of Melbourne Human Research Ethics Committee in November 2018. Recruitment commenced in November 2018 and will be completed in February 2021. The trial is expected to be completed by August 2021 when all participants are due to have completed 6month follow-up.

\section{Discussion}

This will be the first RCT to test the efficacy of footwear for managing pain associated with predominantly lateral TF OA. We hypothesise that motion-control shoes will reduce knee pain more than neutral shoes over 6 months. Footwear is a promising self-management biomechanical treatment for people with knee OA, however all previous research has evaluated footwear in samples of people with predominantly medial TF OA. Findings from these studies cannot be applied to people with lateral TF OA, given that previous research has shown that people with lateral TF OA walk with different biomechanics to those with medial TF OA. There is plausible biomechanical evidence that motion-control shoes may shift load away from the lateral TF compartment, and thus may potentially improve pain, in people with lateral knee OA. Findings from the FOLK clinical trial will provide the first ever evidence about the effects of any type of footwear for people with lateral TF OA, and will thus help inform clinical guidelines about which types of footwear are optimal for managing symptoms in this important subgroup of people with OA.

\section{Abbreviations}

AQoL: Assessment of quality of life; CONSORT: Consolidated standards of reporting trials; FPI: Foot posture index; HREC: Human Research Ethics Committee; KL: Kellgren \& Lawrence; KOOS: Knee injury and osteoarthritis outcome score; NRS: Numerical rating scale; OA: Osteoarthritis; PASE: Physical activity scale for the elderly; RCT: Randomised controlled trial;

SPIRIT: Standard Protocol Items: Recommendations for Intervention Trials;

TF: Tibiofemoral; WOMAC: Western Ontario and McMaster Universities

\section{Acknowledgements}

Not applicable.

\section{Authors' contributions}

$K L P, K L B$ and RSH conceived the idea for the study and RSH is leading the trial. RSH, KLP and JK obtained funding for the study. KLP, KLB, and RSH designed the trial protocol with input from BRM, PKC, JK and TW. KLP drafted the manuscript with input from KLB, BRM, PKC, JK, TW and RSH. The authors read and approved the final manuscript.

\section{Funding}

This study is funded by the National Health and Medical Research Council (Project Grant \#1124418). RSH is supported by a National Health \& Medical Research Council Senior Research Fellowship (\#1154217). The funders have no role in conduct, analysis or reporting of this study.

Availability of data and materials

The datasets used and/or analysed during the current study will be made available from the corresponding author on reasonable request.

Ethics approval and consent to participate

Ethical approval has been obtained from the University of Melbourne Human Research Ethics Committee (HREC No. 1852787.1). All participants will be required to provide written informed consent to participate.

Consent for publication

Not applicable.

Competing interests

$\mathrm{KLP}$ is a member of the Editorial Board of BMC Musculoskeletal Disorders. 


\section{Author details}

'Centre for Health, Exercise and Sports Medicine, Department of Physiotherapy, School of Health Sciences, Faculty of Medicine Dentistry \& Health Sciences, The University of Melbourne, Melbourne, Australia. ${ }^{2}$ School of Public Health and Preventive Medicine, Monash University, Melbourne, Australia.

\section{Received: 11 November 2019 Accepted: 7 April 2020}

\section{Published online: 15 April 2020}

\section{References}

1. James SL, Abate D, Abate KH, Abay SM, Abbafati C, Abbasi N, et al. Global, regional, and national incidence, prevalence, and years lived with disability for 354 diseases and injuries for 195 countries and territories, 1990-2017: a systematic analysis for the global burden of disease study 2017. Lancet. 2018;392:1789-858.

2. Andriacchi TP, Mundermann A. The role of ambulatory mechanics in the initiation and progression of knee osteoarthritis. Curr Opin Rheumatol. 2006; 18:514-8.

3. Chehab EF, Favre J, Erhart-Hledik JC, Andriacchi TP. Baseline knee adduction and flexion moments during walking are both associated with 5 year cartilage changes in patients with medial knee osteoarthritis. Osteoarthr Cartil. 2014;22:1833-9.

4. Schipplein OD, Andriacchi TP. Interaction between active and passive knee stabilizers during level walking. J Orthop Res. 1991;9:113-9.

5. Ledingham J, Regan M, Jones A, Doherty M. Radiographic patterns and associations of osteoarthritis of the knee in patients referred to hospital. Ann Rheum Dis. 1993;52:520-6

6. Fascingbauer M, Renner L, Waldstein W, Boettner F. Are lateral compartment osteophytes a predictor for lateral cartilage damage in varus osteoarthritic knees?: data from the osteoarthritis initiative. Bone Jt J. 2015;97B:1634-9.

7. Cotofana S, Buck R, Wirth W, Roemer F, Duryea J, Nevitt M. al e. cartilage thickening in early radiographic knee osteoarthritis: a within-person, between-knee comparison. Arthritis Care Res. 2012;64:1681-90.

8. Yamabe E, Ueno T, Miyagi R, Watanabe A, Guenzi C, Yoshioka H. Study of surgical indication for knee arthroplasty by cartilage analysis in three compartments using data from osteoarthritis initiative (OAl). BMC Musculoskelet Disord. 2013;14:194.

9. Nagaosa $Y$, Lanyon P, Doherty M. Characterisation of size and direction of osteophyte in knee osteoarthritis: a radiographic study. Ann Rheum Dis. 2002;61:319-24.

10. lijima H, Aoyama T, Nishitani K, Ito H, Fukutani N, Isho T, et al. Coexisting lateral tibiofemoral osteoarthritis is associated with worse knee pain in patients with mild medial osteoarthritis. Osteoarthr Cartil. 2017;25:1274-81.

11. McAlindon TE, Bannuru RR, Sullivan MC, Arden NK, Berenbaum F, BiermaZeinstra SM, et al. OARSI guidelines for the non-surgical management of knee osteoarthritis. Osteoarthr Cartil. 2014;22:363-88.

12. National Clinical Guideline Centre. Osteoarthritis: care and management in adults. Clinical guideline CG177. Methods, evidence and recommendations. London: National Institute for Health and Care Excellence; 2014.

13. Fernandes L, Hagen KB, Bijlsma JWJ, Andreassen O, Christensen P, Conaghan PG, et al. EULAR recommendations for the non-pharmacological core management of hip and knee osteoarthritis. Ann Rheum Dis. 2013;72 $1125-35$.

14. Butler R, Barrios J, Royer T, Davis I. Frontal-plane gait mechanics in people with medial knee osteoarthritis are different from those in people with lateral knee osteoarthritis. Phys Ther. 2011;91:1235-43.

15. Shaw KE, Charlton JM, Perry CK, de Vries CM, Redekopp MJ, White JA, et al. The effects of shoe-worn insoles on gait biomechanics in people with knee osteoarthritis: a systematic review and meta-analysis. Br I Sports Med. 2018; 52:238-53.

16. Paterson KL, Bennell KL, Wrigley TV, Metcalf BR, Kasza J, Hinman RS. Effects of footwear on the knee adduction moment in medial knee osteoarthritis: classification criteria for flat flexible vs stable supportive shoes. Osteoarthritis Cartilage. 2017;25:234-41

17. Bennell KL, Kean CO, Wrigley TV, Hinman RS. Effects of a modified shoe on knee load in people with and without knee osteoarthritis. Arthritis Rheum. 2013;65:701-9.

18. Zafar AQ, Zamani R, Akrami MJG. The effectiveness of foot orthoses in the treatment of medial knee osteoarthritis: a systematic review. Gait Posture. 2019;76:238-51.
19. Kean CO, Bennell KL, Wrigley TV, Hinman RS. Modified walking shoes for knee osteoarthritis: mechanisms for reductions in the knee adduction moment. J Biomech. 2013;46:2060-6.

20. Jenkyn TR, Erhart JC, Andriacchi TP. An analysis of the mechanisms for reducing the knee adduction moment during walking using a variable stiffness shoe in subjects with knee osteoarthritis. J Biomech. 2011:44:1271-6.

21. van Tunen JAC, Paterson KL, Wrigley TV, Metcalf BR, Thorlund JB, Hinman RS. Effect of knee unloading shoes on regional plantar forces in people with symptomatic knee osteoarthritis - an exploratory study. J Foot Ankle Res. 2018;11:34.

22. Radzimski AO, Mundermann A, Sole G. Effect of footwear on the external knee adduction moment - a systematic review. Knee. 2012;19:163-75.

23. Shakoor N, Sengupta M, Foucher KC, Wimmer MA, Fogg LF, Block JA. Effects of common footwear on joint loading in osteoarthritis of the knee. Arthritis Care Res. 2010;62:917-23.

24. Aminian G, Safaeepour Z, Farhoodi M, Pezeshk AF, Saeedi H, Majddoleslam B. The effect of prefabricated and proprioceptive foot orthoses on plantar pressure distribution in patients with flexible flatfoot during walking. Prosthet Orthot Int. 2013;37:227-32

25. Rodrigues P, Ferreira A, Pereira R, Bonfa E, Borba E, Fuller R. Effectiveness of medial-wedge insole treatment for valgus knee osteoarthritis. Arthritis Rheumatism. 2008:59:603-8.

26. Schmalz T, Blumentritt S, Drewitz H, Freslier M. The influence of sole wedges on frontal plane kinetics, in isolation and in combination with representative rigid and semi-rigid ankle-foot-orthoses. Clin Biomech. 2006; 21:631-9.

27. Franz JR, Dicharry J, Riley PO, Jackson K, Wilder RP, Kerrigan DC. The influence of arch supports on knee torques relevant to knee osteoarthritis. Med Sci Sports Exerc. 2008;40:913-7.

28. Rodrigues PT, Ferreira AF, Pereira RMR, Bonfá E, Borba EF, Fuller R. Effectiveness of medial-wedge insole treatment for valgus knee osteoarthritis. Arthritis Care Res. 2008:59:603-8.

29. Chan A, Tetzlaff JM, Altman DG, et al. Spirit 2013 statement: defining standard protocol items for clinical trials. Ann Intern Med. 2013;158:200-7.

30. Altman R, Asch E, Bloch D, Bole G, Borenstein D, Brandt K, et al. Development of criteria for the classification and reporting of osteoarthritis. Classification of osteoarthritis of the knee. Arthritis Rheum. 1986;29:1039-49.

31. Kellgren JH, Jeffrey MR, Ball J. The epidemiology of chronic rheumatism: atlas of standard radiographs. Vol. volume 2. Oxford: Blackwell Scientific; 1963.

32. Culvenor A, Engen C, Øiestad B, Engebretsen L, Risberg MJO. Defining radiographic knee osteoarthritis: a comparison between the Kellgren \& Lawrence classification and oarsi atlas. Cartilage. 2014;22:S265.

33. Altman RD, Gold GE. Atlas of individual radiographic features in osteoarthritis, revised. Osteoarthr Cartil. 2007;15:A1-56.

34. Hinman RS, Wrigley TV, Metcalf BR, Campbell PK, Paterson KL, Hunter DJ, et al. Unloading shoes for self-management of knee osteoarthritis: a randomized trial. Ann Intern Med. 2016;165:381-9.

35. Bellamy N. Osteoarthritis clinical trials: candidate variables and clinimetric properties. J Rheumatol. 1997;24:768-78.

36. Bellamy N. Outcome measurement in osteoarthritis clinical trials. J Rheumatol. 1995;22:49-51.

37. Bellamy N, Buchanan WW, Goldsmith CH, Campbell J, Stitt LW. Validation study of WOMAC: a health status instrument for measuring clinically important patient relevant outcomes to antirheumatic drug therapy in patients with osteoarthritis of the hip or knee. J Rheumatol. 1988:15:1833-40.

38. McConnell S, Kolopack P, Davis AM. The Western Ontario and McMaster universities osteoarthritis index (WOMAC): a review of its utility and measurement properties. Arthritis Rheum. 2001;45:453-61.

39. Roos EM, Roos HP, Lohmander LS, Ekdahl C, Beynnon BD. Knee injury and osteoarthritis outcome score (KOOS)--development of a self-administered outcome measure. J Orthop Sports Phys Ther. 1998;28:88-96.

40. ten Klooster PM, Drossaers-Bakker KW, Taal E, van de Laar MA. Patientperceived satisfactory improvement (PPSI): interpreting meaningful change in pain from the patient's perspective. Pain. 2006;121:151-7.

41. Osborne RH, Hawthorne G, Lew EA, Gray LC. Quality of life assessment in the community-dwelling elderly: validation of the assessment of quality of life (AQoL) instrument and comparison with the SF-36. J Clin Epidemiol. 2003:56:138-47.

42. Martin K, Rejeski W, Miller M, James M, Ettinger W Jr, Messier S. Validation of the PASE in older adults with knee pain and physical disability. Med Sci Sports Exerc. 1999;31:627-33. 
43. Hsu R, Himeno S, Coventry M, Chao E. Normal axial alignment of the lower extremity and load-bearing distribution at the knee. Clin Orthop Relat Res. 1990;255:215-27.

44. Lorig K, Chastain RL, Ung E, Shoor S, Holman HR. Development and evaluation of a scale to measure perceived self-efficacy in people with arthritis. Arthritis Rheum. 1989;32:37-44.

45. Redmond AC, Crosbie J, Ouvrier RA. Development and validation of a novel rating system for scoring standing foot posture: the foot posture index. Clin Biomech. 2006;21:89-98.

46. McPoil T, Vicenzino B, Cornwall M, Collins N, Warren M. Reliability and normative values for the foot mobility magnitude: a composite measure of vertical and medial-lateral mobility of the midfoot. J Foot Ankle Res. 2009;2:6.

47. Brody DM. Techniques in the evaluation and treatment of the injured runner. Orthop Clin North Am. 1982;13:541.

48. Paterson KL, Kasza J, Bennell KL, Wrigley TV, Metcalf BR, Campbell PK, et al. Moderators and mediators of effects of unloading shoes on knee pain in people with knee osteoarthritis: an exploratory analysis of the SHARK randomised controlled trial. Osteoarthritis Cartilage. 2018;26:227-35.

49. Bellamy N, Carette S, Ford P, Kean W, le Riche N, Lussier A, et al. Osteoarthritis antirheumatic drug trials. III. Setting the delta for clinical trialsresults of a consensus development (Delphi) exercise. J Rheumatol. 1992;19: 451-7.

50. Bennell KL, Bowles KA, Payne C, Cicuttini F, Williamson E, Forbes A, et al. Lateral wedge insoles for medial knee osteoarthritis: 12 month randomised controlled trial. BMJ. 2011;342:d2912.

51. Maracy M, Dunn G. Estimating dose-response effects in psychological treatment trials: the role of instrumental variables. Stat Methods Med Res. 2011;20:191-215.

\section{Publisher's Note}

Springer Nature remains neutral with regard to jurisdictional claims in published maps and institutional affiliations.

Ready to submit your research? Choose BMC and benefit from:

- fast, convenient online submission

- thorough peer review by experienced researchers in your field

- rapid publication on acceptance

- support for research data, including large and complex data types

- gold Open Access which fosters wider collaboration and increased citations

- maximum visibility for your research: over $100 \mathrm{M}$ website views per year

At $\mathrm{BMC}$, research is always in progress.

Learn more biomedcentral.com/submissions 